\title{
Erratum to: Subgroups Analysis when Treatment and Moderators are Time-varying
}

\author{
Daniel Almirall • Daniel F. McCaffrey • \\ Rajeev Ramchand • Susan A. Murphy
}

Published online: 11 October 2012

(C) Society for Prevention Research 2012

\section{Erratum to: Prev Sci}

\section{DOI 10.1007/s11121-011-0208-7}

The development of this article was funded by the following grants: R01DA015697 (PI: McCaffrey), R01MH080015 (PI: Murphy), and P50DA010075 (PI: Murphy).

It was also supported by the Center for Substance Abuse Treatment (CSAT), Substance Abuse and Mental Health Services Administration (SAMHSA) contract \#270-07-0191 using data provided by the following grantees: Cannabis Youth Treatment (Study: CYT; CSAT/SAMHSA contracts \#270-97-7011, \#270-00-6500, \#270-2003-00006 and grantees: TI-11317, TI-11321, TI-11323,TI-11324), Adolescent Treatment Model (Study: ATM: CSAT/SAMHSA contracts \#270-98-7047, \#270-97-7011, \#277-00-6500, \#270-2003-
00006 and grantees: TI-11894, TI-11892, TI-11422, TI11423, TI-11424, TI-11432), the Strengthening Communities Youth (Study: SCY; CSAT/SAMHSA contracts \#27700-6500, \#270-2003-00006 and grantees: TI-13344, TI13354, TI-13356), and Targeted Capacity Expansion (Study: TCE; CSAT/SAMHSA contracts \#270-200300006, \#270-2007-00004C, and \#277-00-6500 and grantee TI-16400). The authors thank these grantees and their participants for agreeing to share their data to support the secondary data analysis to illustrate the methodology. The opinions about this data are those of the authors and do not reflect official positions of the government or individual grantees. Please direct correspondence to Daniel Almirall, PhD, dalmiral@umich.edu, 734-9363077.

The online version of the original article can be found at http://dx.doi.org/ 10.1007/s11121-011-0208-7.

\section{Almirall $(\bowtie)$}

Institute for Social Research, University of Michigan,

426 Thompson Street, Suite 2204, Ann Arbor,

MI 48104-2321, USA

e-mail:dalmiral@umich.edu

D. F. McCaffrey

RAND Corporation's Pittsburgh Office,

Pittsburgh, PA, USA

R. Ramchand

RAND Corporation's Washington, DC Office,

Washington, DC, USA

S. A. Murphy

Department of Statistics and Institute for Social Research,

University of Michigan, 439 West Hall, 1085 South

University Ave., Ann Arbor, MI 48109-1107, USA 